\title{
Intermanual Transfer of the Curvature Aftereffect in Static and Dynamic Touch
}

\author{
Bernard J. van der Horst and Astrid M.L. Kappers \\ Utrecht University, Helmholtz Institute \\ Princetonplein 5, 3584 CC Utrecht, The Netherlands \\ b.j.vanderhorstaphys.uu.nl
}

\begin{abstract}
The existence and intermanual transfer of curvature aftereffects was studied for static and dynamic touch, whereby only the index fingers were used. A curvature aftereffect is the phenomenon that a flat surface is judged concave if the preceding touched stimulus was convex and vice versa. Substantial aftereffects were demonstrated when the subsequently presented adaptation and test stimulus were touched by the same index finger. When one index finger was used to touch the adaptation stimulus and the opposite index finger was employed to touch the test stimulus, only a partial transfer of the aftereffect was found for static touch, but a complete transfer was obtained in the dynamic case. These findings suggest that the representation of curvature information depends on the exploration mode.
\end{abstract}

Keywords: Aftereffect - Intermanual Transfer - Curvature - Haptic - Touch.

\section{Introduction}

When you touch a flat surface following the prolonged touching of a convex surface, the flat surface feels concave. Likewise, the flat surface seems to be convex when the preceding contacted surface was concave [1]. This phenomenon is called a haptic curvature aftereffect (see Fig. 1). This effect pertains to a broader class of aftereffect phenomena, which have in common that the perception of a physical property is systematically altered by preceding obtained stimulation of that property. Haptic aftereffects have been demonstrated, for example, for the perception of motion information on the arm [2] or finger [3], for the perception of the size of an object when it is grasped by two fingers [4], or for the judgment of the vergence of two surface in space with the palms of the hands [5].

Haptic curvature aftereffects have been found for static and dynamic touch. A $d y$ namic curvature aftereffect was reported when a curved edge was explored by to and fro movement of the fingers. Adaptation time was three minutes [6]. A static curvature aftereffect was demonstrated when spherically curved surfaces were touched by the whole hand. The aftereffect was measured by presenting a test surface following 10 seconds of adaptation [1]. It has not been investigated whether a static aftereffect exists when curved surfaces are touched by only a single fingertip. Furthermore, it is 


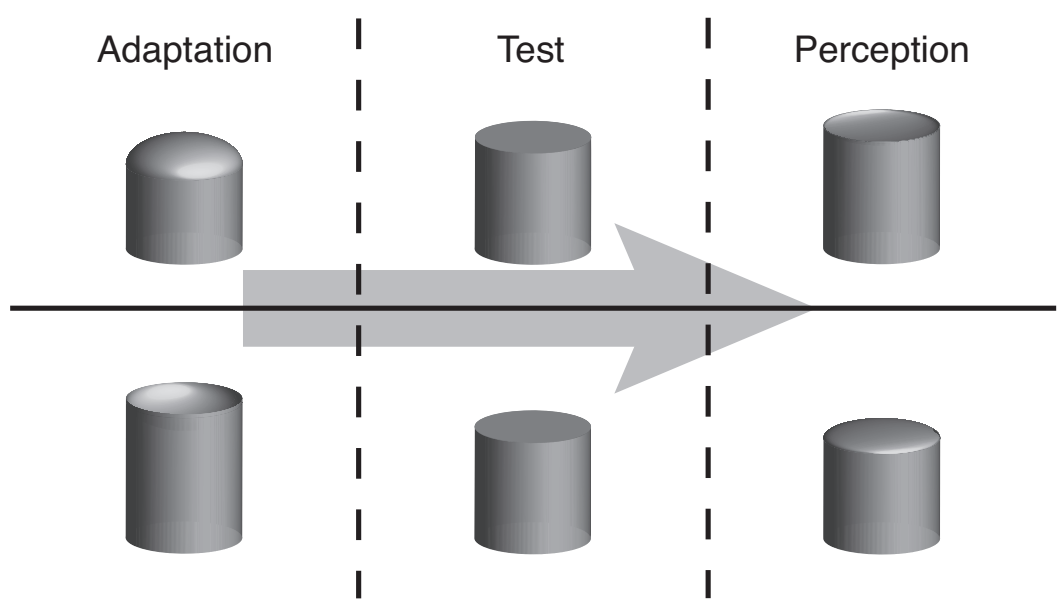

Fig. 1. Schematic overview of a haptic curvature aftereffect: when you first touch a convex (concave) surface for 10 seconds and subsequently touch a flat surface, this latter surface feels concave (convex).

unknown whether a dynamic aftereffect occurs when the exploration mode is restrained to a single finger and the adaptation time is restricted to only ten sec. The first purpose of this study was to assess the occurrence of static and dynamic curvature aftereffects, when curved surfaces are touched by a single finger.

The second purpose of this study was to investigate whether the aftereffect transfers between the fingers of both hands. Transfer of the aftereffect means that adaptation with one finger generates a change in the perception of another finger. Probing the transfer characteristics of the aftereffect is interesting, as it reveals insight into the representation of a perceived property. For this reason, in vision, the transfer of the aftereffect from one eye to the other eye has been studied extensively [7], [8]. Similarly, establishing the transfer characteristics of the haptic curvature aftereffect would provide insight into the representation of curvature information. For the transfer of the aftereffect, we could formulate three hypotheses in advance. The first hypothesis is that there is no intermanual transfer. This implies that each hand has its own, separate representation of curvature information. The second hypothesis is that there is a complete, intermanual transfer. This entails that curvature is not processed for each finger or hand separately, but is represented at a common, bilateral level. The third hypothesis is that there is a partial transfer. There is not a single level on which curvature information is processed, but a part of the representation is formed at a degree directly connected to a single finger and a part of the representation is a bilateral stage. Note that these three hypotheses are formulated for haptic aftereffect transfer in general. The transfer characteristics are not necessarily similar for static touch and for dynamic touch. A difference in the extent of the transfer would indicate that the representation of curvature depends on the mode of exploration. 


\section{Methods}

\subsection{Static Touch}

\subsubsection{Stimuli}

The stimuli were made of a synthetic material (Cibatool BM 5460). A computer controlled milling machine was used to produce cylinders with a flat bottom and a spherically curved top. The top was either pointing outward (convex) or inward (concave). A convex and concave adaptation stimulus was used. The curvature of these stimuli was +36 and $-36 \mathrm{~m}^{-1}$, respectively. Nine test stimuli were used, with the curvature ranging from $-16 \mathrm{~m}^{-1}$ to $+16 \mathrm{~m}^{-1}$, in steps of $4 \mathrm{~m}^{-1}$. An illustration of a convex stimulus is given in Fig. 2A.

\subsubsection{Procedure}

Subjects sat behind a table. Their arms rested on a platform, which was $30 \mathrm{~mm}$ above the tabletop. Only the fingertips stuck out from the platform. The experimenter placed the stimuli on the table underneath the fingertips. A curtain prevented the subjects from seeing the stimuli. During a trial, a subject placed the fingertip of the index finger of the preferred hand on an adaptation stimulus for 10 seconds. The start and end of the adaptation period were indicated by a short beep. Subsequently, a test stimulus was placed underneath either the same finger (same-finger condition) or the opposite index finger (transfer condition). The task of the subject was to judge whether the test stimulus felt convex or concave. Generally, the decision was made within a few seconds. The subject was not allowed to move the finger over the stimulus surface. No feedback was provided.

A complete condition was measured by performing 180 trials (two adaptation stimuli $\mathrm{x}$ nine test stimuli $\mathrm{x} 10$ repetitions). The order within each group of 18 trials was randomized. The measurement time was about one and a half hours per subject, who performed a single condition.

\subsubsection{Analysis}

For each subject, the data were analyzed separately for the convex and the concave adaptation stimuli. The fraction of "convex" responses was plotted against the curvature of the test stimulus. A psychometric function (cumulative Gaussian) was fitted to

A

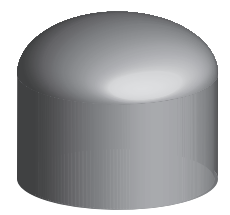

B

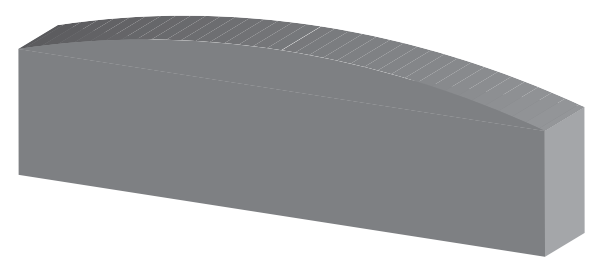

Fig. 2. A Illustration of a convex stimulus, used for static touch. The top of the stimulus is spherically curved. The base of the stimulus is a cylinder, with a radius of $15 \mathrm{~mm}$. The distance between top and bottom is $30 \mathrm{~mm}$. B Illustration of a convex stimulus, used for dynamic touch. The top of the stimulus is cylindrically curved. The base of the stimulus is a rectangular block. The length is $200 \mathrm{~mm}$; the width is $20 \mathrm{~mm}$. The height at the side of the stimulus is $40 \mathrm{~mm}$. 


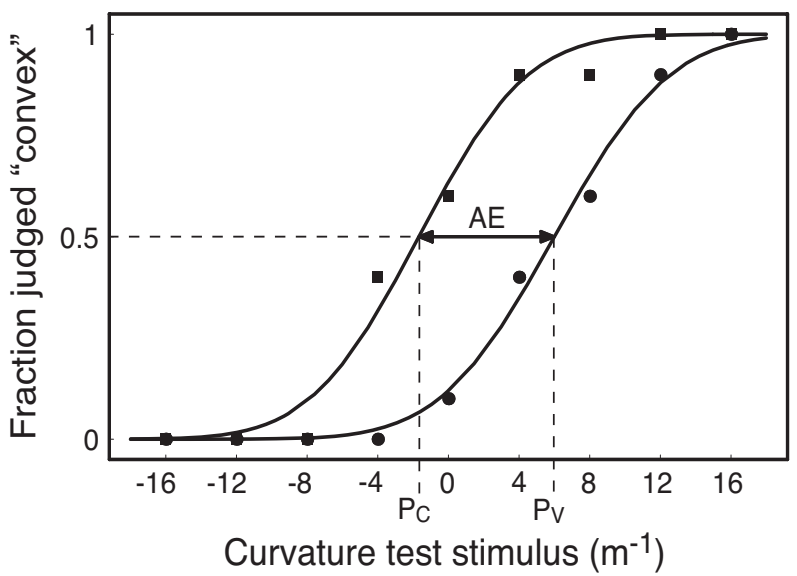

Fig. 3. Examples of two psychometric curves. The circular data points and the fit through these points results from adaptation to the convex adaptation stimulus. The point of subjective equality (PSE) is represented by $\mathrm{P}_{\mathrm{V}}$. The square data points and the fit trough these points result from adaptation to the concave adaptation stimulus. In this case, the PSE is represented by $P_{C}$. The magnitude of the aftereffect is defined as the difference between $\mathrm{P}_{\mathrm{V}}$ and $\mathrm{P}_{\mathrm{C}}$.

the data points in order to determine the point of subjective equality (PSE). The PSE represents the curvature value that in $50 \%$ of the cases was judged "convex". The aftereffect is defined as the difference between the PSE resulting from convex adaptation and the PSE resulting from concave adaptation. Fig. 3 shows psychometric curves for a convex and a concave adaptation. The PSEs and the magnitude of the aftereffect are indicated.

\subsubsection{Subjects}

Eight subjects participated in the same-finger condition (five male and three female, mean age 22 years). Six were right-handed and two were left-handed, according to a standard questionnaire [9]. Sixteen, right-handed subjects (eight male and eight female, mean age 22 years) participated in the transfer condition. None of the subjects participated in both conditions.

\subsection{Dynamic Touch}

\subsubsection{Stimuli}

The stimuli were made of PVC. The shape of the top surface was circularly curved. The curvatures of the adaptation stimuli were +3.8 and $-3.8 \mathrm{~m}^{-1}$. The curvature of the test stimuli ranged from -1.8 to $+1.8 \mathrm{~m}^{-1}$, in steps of $0.4 \mathrm{~m}^{-1}$. An illustration of a stimulus is given in Fig. $2 \mathrm{~B}$.

\subsubsection{Procedure}

The stimuli were placed in a holder on the table, in front of the subject. Subjects were instructed to explore an adaptation stimulus by making sideward movements with the fingertip over the stimulus surface. They had to perform three complete to and fro 
movements, in about 10 seconds. A test stimulus was explored by only a single side to side movement, which took 1 to 2 seconds. Again, the task was to judge whether the test stimulus felt convex or concave. instruct

\subsubsection{Subjects}

Eight, new, right-handed subjects participated in both conditions (four male and four female, mean age 21 years).

\section{Results}

\subsection{Static Touch}

The mean results for the aftereffect are given in Fig. 4A. The error bars represent the standard error in the mean for each condition. The occurrence of an aftereffect was tested by performing separate 1-tailed $t$-tests. A significant result was obtained for both conditions $\left(t_{7}=6.3, p<0.001\right.$ for the same-finger condition and $t_{15}=2.7, p=0.009$ for the transfer condition). The magnitude of the aftereffect in the same-finger condition was significantly higher than in the transfer condition, as was confirmed by an independent samples $t$-test $\left(t_{22}=5.0, p<0.001\right)$.

\subsection{Dynamic Touch}

The mean results are given in Fig. 4B. A significant aftereffect was found in both conditions $\left(t_{7}=5.4, p<0.001\right.$ for the same-finger condition and $t_{7}=4.2, p=0.002$ for the
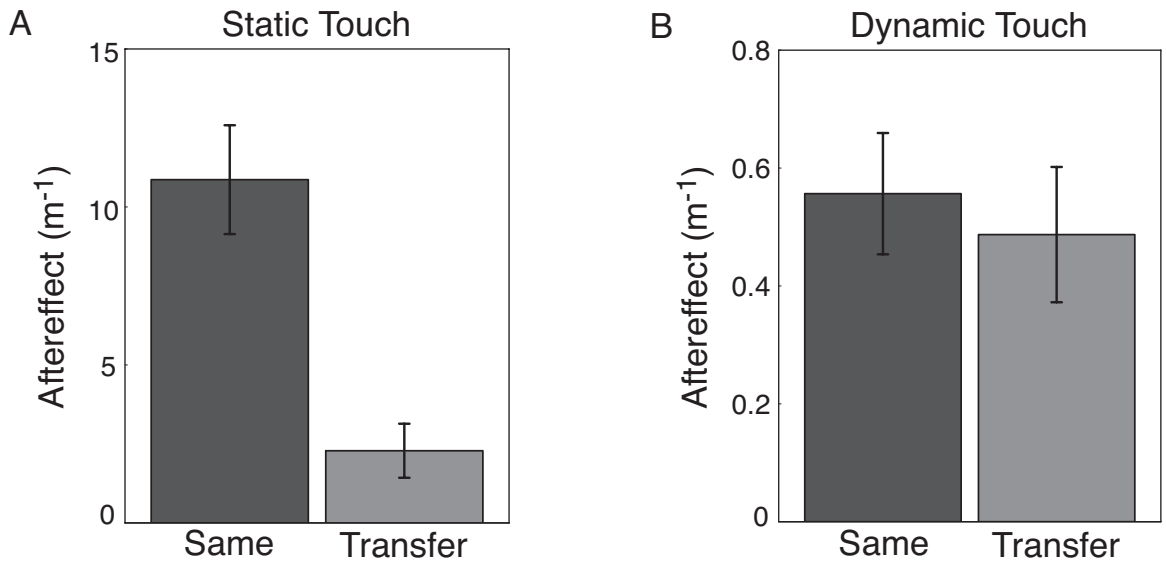

Fig. 4. Mean results for the aftereffect. The error bars represent the standard errors in the mean. The dark bars are the results for the same-finger condition, in which the adaptation and test stimulus are touched by the index finger of the preferred hand. The light bars are the results for the transfer condition, the condition in which the adaptation stimulus was touched by the index finger of the preferred hand and the test stimulus was touched by the non-preferred index finger. 
transfer condition). There was no significant difference in the magnitude of the aftereffect between both conditions, as a dependent samples $t$-test showed $\left(t_{7}=0.4, p=0.7\right)$.

\section{Discussion}

Previous studies have demonstrated that curvature aftereffects occurred when a curved edge was explored [6] or when the whole hand was placed on curved shapes [1], [10]. In this study, we demonstrated the existence of two, novel curvature aftereffects. First, a static aftereffect, when curved adaptation and test surfaces are touched by a single fingertip. Second, a dynamic aftereffect, when curved surfaces are explored by the movement of a single index finger, for a relatively short adaptation time.

Intermanual transfer of the aftereffect was found for static touch and for dynamic touch. These findings differ from the study of Vogels and colleagues [10], who did not find an intermanual transfer. However, the exploration mode was different in their experiment (the whole hand was involved) and the number of subjects was small (only two participants).

The extent of transfer was different for static touch than for dynamic touch. In the static case, the transfer was only partial: the aftereffect was much smaller in the transfer condition than in the same-finger condition. This suggests that an important part of the representation of the curvature information was at a stage that is directly connected to the finger or the hand. Only a small part of the representation was at a level that the fingers of both hands share in common. In the dynamic case, a complete transfer was found, as a similar magnitude for the aftereffect was found in both conditions. This finding suggests that curvature information obtained with one hand is not separated from curvature information acquired with the other hand, but indicates that dynamically perceived curvature is completely represented at a common, bilateral level.

These findings show that the representation of curvature depends on the manner in which this information is received. This result is surprising, as Pont and colleagues found similar curvature discrimination thresholds for static and dynamic touch [11]. However, finding similar thresholds does not necessarily entail that similar processes are involved in the representation of curvature by static or dynamic touch.

At this stage, it is interesting to consider how the processing that is required to perceive shape can be related to the manner of exploration. In static touch, the shape of an object might be deduced from the indentation profile of the finger, as registered by the cutaneous mechanoreceptors. This information is instantaneously available. In contrast, in dynamic touch, the instantaneous indentation profile is insufficient to perceive the shape of the surface. However, other cues are available, when the finger moves over a curved surface. Informative cues are the stretch of the skin, the change in contact point between the finger and the surface, the rotation of the finger, and the vertical displacement of the finger. Perceiving curvature by dynamic touch needs an integration over time of several cues. This process is quite more complex than perceiving curvature by static touch. This might explain the difference in representation level for static and dynamic touch. 
Acknowledgements. This research was supported by a grant from the Netherlands Organization for Scientific Research (NWO).

\section{References}

1. Vogels, I.M.L.C., Kappers, A.M.L., Koenderink, J.J.: Haptic After-effect of Curved Surfaces. Perception 25, 109-119 (1996)

2. Thalman, W.A.: The Aftereffect of Movement in the Sense of Touch. American Journal of Psychology 33, 268-276 (1922)

3. Watanabe, J., Hayashi, S., Kajimoto, H., Tachi, S., Nishida, S.: Tactile Motion Aftereffects Produced by Appropriate Presentation for Mechanoreceptors. Experimental Brain Research 180, 577-582 (2007)

4. Koehler, W., Dinnerstein, D.: Figural After-effects in Kinesthesis. In: Institut Superieure de Philosophi (ed.) Miscellanea Psychologica Albert Michotte. Imprimerie St-Alphonse, Louvain, pp. 196-220 (1947)

5. Gibson, J.J., Backlund, F.A.: An After-effect in Haptic Space Perception. Quarterly Journal of Experimental Psychology 15, 145-154 (1963)

6. Gibson, J.J.: Adaptation, After-effect and Contrast in the Perception of Curved Lines. Journal of Experimental Psychology 16, 1-31 (1933)

7. Moulden, B.: After-effects and the Integration of Patterns of Neural Activity within a Channel. Phil. Trans. R. Soc. Lond. B. 290, 39-55 (1980)

8. Wade, N.J., Swanston, M.T., de Weert, C.M.M.: On Interocular Transfer of Motion Aftereffects. Perception 22, 1365-1380 (1993)

9. Coren, S.: The Left-Hander Syndrome. Vintage Books, New York

10. Vogels, I.M.L.C., Kappers, A.M.L., Koenderink, J.J.: Investigation into the Origin of the Haptic After-effect of Curved Surfaces. Perception 26, 101-117 (1997)

11. Pont, S.C., Kappers, A.M.L., Koenderink, J.J.: Similar Mechanisms Underlie Curvature Comparison by Static and Dynamic Touch. Perception \& Psychophysics 61, 874-894 (1999) 